\title{
The detection of Escherichia coli biofilm in green oak with spectroscopic imaging techniques based on confocal laser scanning microscope
}

\author{
Jintaphorn Klinsuk ${ }^{1}$, Natphichon Budtri ${ }^{1}$, Suwan Aekrum ${ }^{1}$, Pattarapong Sunongbua ${ }^{1}$, \\ Charoonsak Somphong ${ }^{2}$, and Weerasak Lertsiriyothin..$^{1}$. \\ 1,2 Suranaree University of Technology, Institute of Engineering, School of Agricultural Engineering \\ 30000. Nakhonratchasima, THIALAND
}

\begin{abstract}
Microbial recontamination in post-harvest products, specifically for fresh cut fruits and vegetables, often raises concern of consumer health safety since pathogen bacteria readily forms sticky biofilm for their protection that often render ineffective of cleaning or disinfection process. Therefore, tracking biofilm rather than bacteria cells may also be used for indication of microbial recontamination. This research focuses on acquisition of spectroscopic imaging and information for E. coli biofilm adherent on green oak leaf at microscopic level for visible bandwidth. A confocal laser scanning microscope (CLSM) was applied to investigate for the evident of biofilm formation. In order to enhance spectroscopic signal, staining surface sample with propidium iodide, commonly used for staining dead cell, was specifically investigated to a potential use for determination of E.coli's biofilm as an evident of recontamination occurrence. Green oak leaf samples were inoculated with a small drop of viable E. coli of $2.7 \times 10^{9}$ $\mathrm{CFU} / \mathrm{ml}$ covering around $7 \times 7 \mathrm{~mm}^{2}$ and all samples were spectroscopic imaged every day for 7 days. Absorbance spectrum acquired by CLSM for the $E$. coli inoculated samples presented three identity peaks at wavelengths of 620,670 , and $690 \mathrm{~nm}$ but the latter identity wavelength appeared to include common autofluorescence of leaf surface physiology so arguable interpretation is unavoidable for $690 \mathrm{~nm}$. In opposite, the identity peaks at 620 and $670 \mathrm{~nm}$ presented strong detection of $E$. coli 's biofilm for storage time beyond day 5 at a significant level of confident $99 \%$. However, the lowest minimum detection limit in term of E. coli concentration required more precisely experimental validation.
\end{abstract}

Keyword: Escherichia coli, Biofilm, CLSM, Spectroscopic

*Corresponding author :LSRYTW@g.sut.c.th 


\section{Introduction}

Biofilm is a matrix of exopolysaccharides, protein, DNA and some of lipid produced by bacterial cells that attached on the surface of various substrates and may be called as extracellular polymeric substance (EPS) [1]. The bacterial cells with biofilm have high resistance to disinfectant, then consumption of cross contamination in food products can be harmful to consumers. Consequently, biofilm detection methods was indeed useful for food safety and quality inspection.

Since 1990, there have been several studies about biofilm formation and microbial growing rate using spectroscopic technique by determining the strains absorbance value with crystal violet staining [2]. Subsequently, the technique of biofilm examination was developed by combining spectroscopic and imaging. Brider et al. [3] used the Syto9 for DNA stain and live/dead cell stains including analysis responsibility with a confocal laser scanning microscope ( CLSM) in 2010. CLSM analysis becomes a popular tool for study of microorganism and biofilm. By virtue of the staining reagents, CLSM can provide both image and specific reflective spectra correspondent with absorbance wavelength. Therefore, many researchers have been characterize biofilm produced by various bacteria based on their specific spectrum. Kim et al. [4] reported that the biofilm formation of E. coli occurred on several types of surface materials, namely HDPE, Formica-type plastic laminate, Granite uniform black color, granite non-uniform, and stainless steel and its major spectrum was characterized at wavelength of $470 \mathrm{~nm}$. Rodney 2002 [1] reported that dapi maker was a good staining reagent for biofilm with a specific wavelength of $450 \mathrm{~nm} .$. In 2017, Chow goon $\mathrm{Ng}$ et al [6] used antibody markers, Alexa Fluor-tagged goat and anti-rabbit antibodies and scanning electron microscope to investigate the biofilm formation on vegetables. The vegetables without cultures always have the response of autofluorescence at green and red colors (550-700 nm approximately) while biofilm formation on the vegetables with culture responded to specific wavelength dependent on the antibody markers.

The purposes of this research was to reinvestigate whether propidium iodide can be used to characterize biofilm formed by E. coli on the surface of green oak salad. A confocal laser scanning microscope (CLSM) was applied to identify the identity of absorbance wavelength responsible for E. coli's biofilm stained by propidium iodide. Spectroscopic imaging technique was proposed for risk evaluation of green oak salad consumption.

\section{Materials and methods}

The culture growing reagent such as Nutrient Agar,,Trypic soy Broth was purchased form Sigma Aldrich .For the 1XPBS and Propidium Iodide staining reagent was purchased from Invitrogen Molecular Probes( UK).

\subsection{Samples preparation}

The green oak was purchased from Suranaree University of Technology farm, Thailand. Samples were washed with DI water and added $1000 \mathrm{ml}$ of $100 \mathrm{ppm}$ latron CS7-100 for 30 min for decreasing a surface tension on green oak leaves. The samples were washed with DI water and dried at ambient conditions. 


\subsubsection{Biofilm formation on green oak}

Escherichia Coli TISTR371 was received form Biodiversity Research Center, Institute of Scientific, Thailand. It was first plated on nutrient agar (Sigma Aldrich) and incubated at $37^{\circ} \mathrm{C}$ for $24 \mathrm{hrs}$. Single colonies were transferred to $10 \mathrm{ml}$ of Trypic soy broth then incubated at $37^{\circ} \mathrm{C}$ for $24 \mathrm{hrs}$. After $24 \mathrm{hrs}, 10 \mu \mathrm{l}$ of culture was dropped on green oak leaves at each position then incubated at $8^{\circ} \mathrm{C}$ for 5 days. The sample was observed inspected at $0,1,3,5$ and 7 days, respectively.

\subsubsection{Confocal laser scanning microscope (CLSM)}

The samples with biofilm formation process were rinsed with DI water for removing nonadherent bacteria. Then, $50 \mu 1$ of propidium iodide (PI) staining was dropped on the leaves and incubated in dark at room temperature for 30 minutes. The sample were washed with DI sterile for cleaning non stained reagent out of surface. The leaves were cut and placed on glass slide. Then, they were analyzed by using Nikon $80 \mathrm{E}$ inverted Confocal Laser Scanning Microscope in confocal mode and spectral mode with laser excitation/emission at 488/500$550 \mathrm{~nm}$ and $561 / 570-620 \mathrm{~nm}$.

\subsubsection{Scanning electron microscopy (SEM)}

The sample preparation process for SEM was shown in Figure 1. The freeze vegetable and culture cell sample with different conditions were cut to $1 \times 1 \mathrm{~cm}^{2}$ and soaked in $2.5 \%$ of glutaraldehyde in $0.5 \times \mathrm{PBS}(\mathrm{pH} 7.2$ ). Then, the samples were washed 3 times with $0.5 \mathrm{xPBS}$. After washing process, the samples were incubated in 1\% osmium tetroxide for $2 \mathrm{hrs}$ and washed with sterile DI water 3 times. The samples were dehydrated with acetone sequentially ( $20 \%, 40 \%, 60 \%, 80 \%$, and $100 \%$ of acetone). After dehydration process, samples were dried by critical point dryer. The morphology of biofilm and bacteria cells were analysed by FIB-SEM Carl Zeiss Model Auriga.

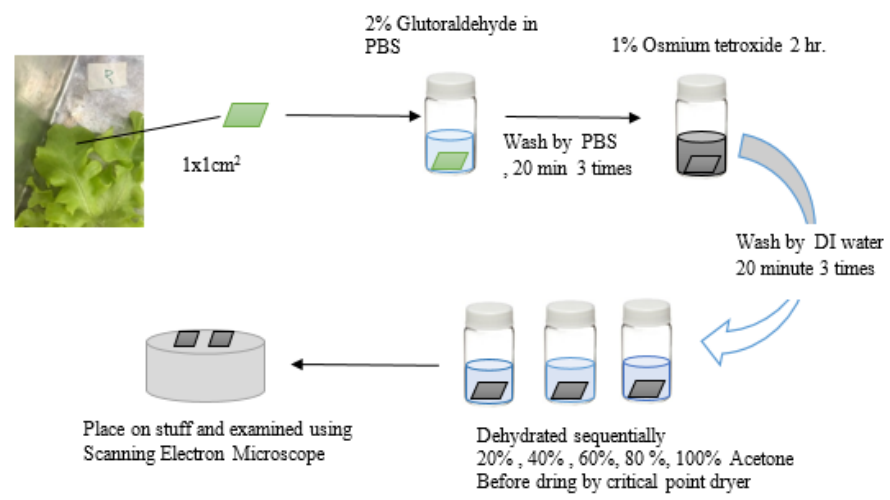

Fig. 1. The sample preparation process for Scanning Electron Microscope. 


\section{Results and discussion}

\subsection{Confocal laser scanning microscope}

Evident of E. coli and its' biofilm formation on the leaf surface of green oak salad was characterized by using propidium iodide staining technique and CLSM imaging under a confocal mode at 488 and $561 \mathrm{~nm}$ excitation. Selective green and red color channels of CLSM images of no culture area without PI staining (Figure 2A), no culture area with PI staining (Figure 2B), and E coli doped-area with PI staining (Figure 2C) showed that stomata of control samples regardless of PI staining clearly appeared in green, but little or no emission in red as shown in Figure 2A and 2B [6]. However, a selective wavelength (690 nm) image of no culture area with PI staining showed dominant red color, but no red image was noticeable at the other two red wavelengths of 620 and $670 \mathrm{~nm}$ as seen in the case of $E$ coli doped-area with PI-staining (Figure 3). The spectral signal at 680-690 nm represented the chloroplast with chlorophyll A. The Chlorophyll A was commonly existed in green plants and emitted at wavelength 688 and $730 \mathrm{~nm}$ after being excited by blue-green fluorescence [7-9]. Therefore, the spectral wavelength of 620 and $670 \mathrm{~nm}$ can be used as unambiguous indicators for either E. coli or its' biofilm.

Since biofilm produced from E. coli doped on the green oak leaf is unable to respond to fluorescence excitation, propidium iodide staining reagent was used to enhance the biofilm culture intensity in this case. Propidium iodide is the DNA staining reagent which responds to $535 \mathrm{~nm}$ excitation with $617 \mathrm{~nm}$ emission. Figure $2 \mathrm{C}$ showed that the leaf with culture staining has high intensity than control culture. The rod cell of E. coli was dispersedly found around this area. The red color fluorescence of green oak indicated the emission of propidium iodide staining.

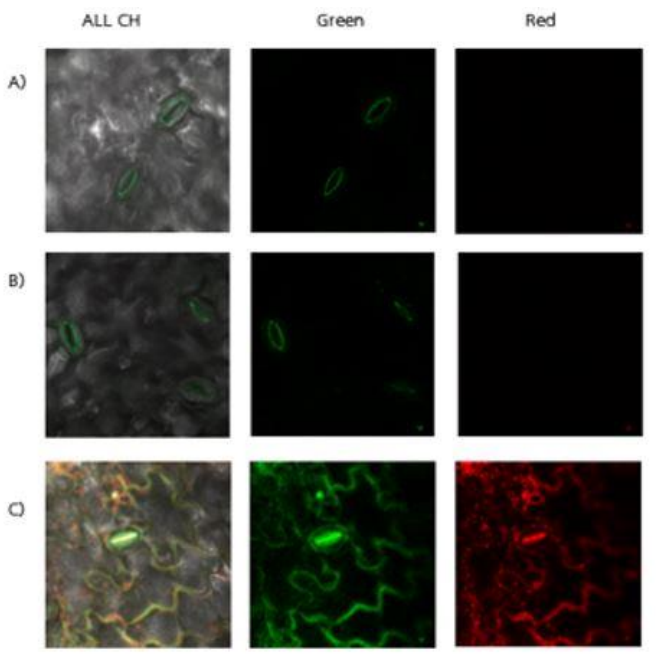

Fig. 2. CLSM images of green oak leaf samples with a magnificent of 40x A) No-culture area without PI staining B) No-culture area with PI staining C) E coli doped-area with PI staining. 


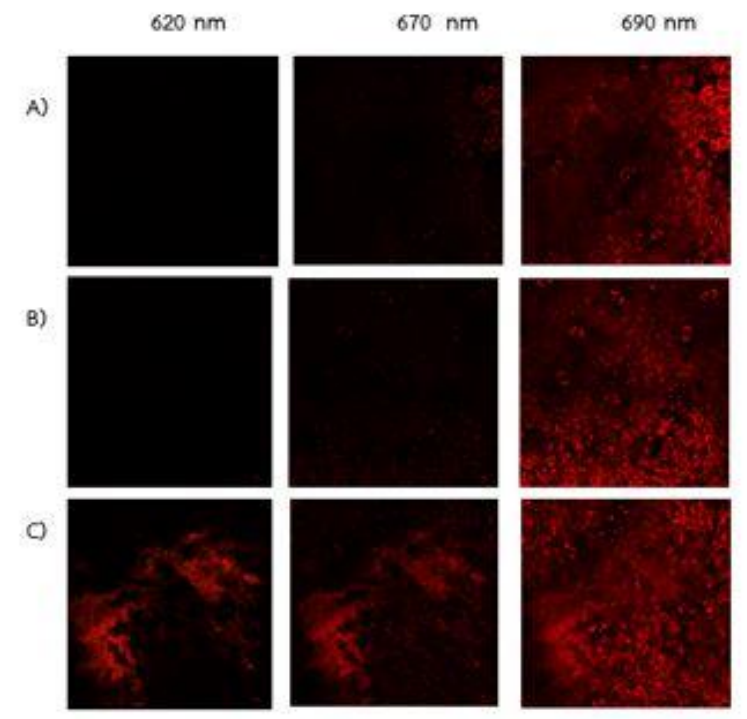

Fig. 3. Three selective wavelengths of CLSM images of green oak leaf samples with a magnificent of 40x A) No-culture area without PI staining B) No-culture area with PI staining C) $E$ coli dopedarea with PI staining.

The spectral of red color from plant auto fluorescence and PI emission were compared in CLSM spectral mode (Figure 3). All of the samples' response was presented at range 670$690 \mathrm{~nm}$ and range 680-690 nm similar to the report of Krause et al. and Kodama [7-8]. On the other hand, A sample of culture stained would respond at a strong wavelength of 620-630 $\mathrm{nm}$ correlated with the culture with PI straining which was presented the fluorescence emission at $617 \mathrm{~nm}$ and the wavelength may shift for about $\pm 3 \mathrm{~nm}$.

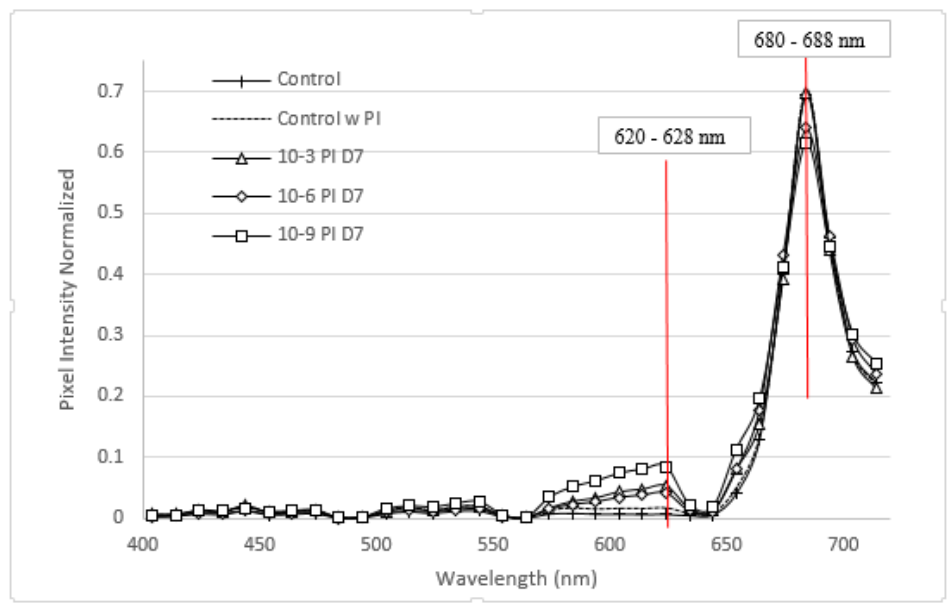

Fig. 4. The normalized spectral profiles of green oak salad for control sample without PI staining, control sample with PI staining and culture dilution with PI staining.

In Figure 4 , the existence of plant autofluorescence and biofilm were substantiated by PI staining. The autofluorescence of vegetables commonly found around 680-690 nm. The results presented that the autofluorescence between green oaks with and without culture was indifferentiable. 
The autofluorescence signal of stomata area was stronger than another area related to chlorophyll content. Nayara in 2013 [9] reported the leaves area with high stomatal index and stomatal density ( stomata/ $\mathrm{mm}^{2}$ ) will induce the high chlorophyll .Morever, chlorophyll level depends on the stomatal conductance. Sami et al [10] describe the effect of stomata on chlorophyll content in the leaf. The area with high stomatal index but low stomatal conductance will make the low chlorophyll content. Therefore, the signal intensity of autofluorescence at 670-680 $\mathrm{nm}$ might not equal depends on analytical areas with stomata index and stomatal conductance.

The biofilm classification of samples were indicated in the spectrum. The pixel intensity was normalized and analysed by $\mathrm{T}$-test analysis and the results presented in Table 1 . The pixel intensity at $620 \mathrm{~nm}$ is different from at day 5 to day 7 at significant 0.1 . On the other hand, control, control with PI staining at $620 \mathrm{~nm}$, and all samples at $680 \mathrm{~nm}$ are not different at $90 \%$. This technique has advantages that it is non-destructive test, provide a large area, and take a shorter time than the convention method (Table 2.). However, the limitation of this method cannot detect the biofilm from day 1 to day 3 because the biofilm formation is low concentration.

Table 1. Normalized spectral intensity to classified Green oak with E. coli biofilms.

\begin{tabular}{|l|r|r|c|c|}
\hline \multirow{2}{*}{ Sample } & \multicolumn{2}{|c|}{$620 \mathrm{~nm}$} & \multicolumn{2}{c|}{$680 \mathrm{~nm}$} \\
\cline { 2 - 5 } & Day 5 & Day 7 & Day 5 & Day 7 \\
\hline Control without PI & $0.009 \pm 0.003^{\mathrm{ab}}$ & $0.007 \pm 0.02^{\mathrm{ab}}$ & $0.610 \pm 0.06^{\mathrm{ab}}$ & $0.696 \pm 0.016^{\mathrm{ab}}$ \\
\hline Control with PI & $0.011 \pm 0.005^{\mathrm{ab}}$ & $0.017 \pm 0.01^{\mathrm{ab}}$ & $0.552 \pm 0.04^{\mathrm{ab}}$ & $0.703 \pm 0.025^{\mathrm{ab}}$ \\
\hline $10^{-6} \mathrm{PI}$ & $0.023 \pm 0.01^{\mathrm{aa}}$ & $0.042 \pm 0.01^{\mathrm{aa}}$ & $0.601 \pm 0.06^{\mathrm{ab}}$ & $0.639 \pm 0.033^{\mathrm{ab}}$ \\
\hline $10^{-9}$ PI & $0.023 \pm 0.08^{\mathrm{aa}}$ & $0.048 \pm 0.004^{\mathrm{aa}}$ & $0.671 \pm 0.03^{\mathrm{ab}}$ & $0.616 \pm 0.060^{\mathrm{ab}}$ \\
\hline
\end{tabular}

aa= has different at significant 0.1 .

$\mathrm{ab}=$ has not different at significant 0.1 .

*Biofilm formation on green oak at low concentration (day 0-day 3) has no different with control samples.

Table 2. Comparison of biofilm detection between convention methods and spectroscopic techniques.

\begin{tabular}{|l|c|c|c|}
\hline \multicolumn{1}{|c|}{ Methods } & Sample charecterization & Testing time & references \\
\hline $\begin{array}{l}\text { Tissure culture plate } \\
\text { (TCP) }\end{array}$ & $\begin{array}{c}\text { Size reduction by } \\
\text { stomacher, dilution and } \\
\text { staind by Crystal violet }\end{array}$ & $24 \mathrm{hr}$ & Pragyan et al [1] $]$ \\
\hline $\begin{array}{l}\text { Tube method } \\
\text { (TM) }\end{array}$ & $\begin{array}{c}\text { Size reduction by } \\
\text { stomacher, dilution and } \\
\text { staind by Crystal violet }\end{array}$ & $24 \mathrm{hr}$ & \\
\hline $\begin{array}{l}\text { Congo Red Agar } \\
\text { (CRA) }\end{array}$ & $\begin{array}{c}\text { Size reduction by } \\
\text { stomacher, dilution }\end{array}$ & $24-48 \mathrm{hr}$ & \\
\cline { 1 - 3 } $\begin{array}{l}\text { Spectroscopic } \\
\text { techniques }\end{array}$ & Surface & $\begin{array}{c}1 \mathrm{hr} \text { up (depend on } \\
\text { concentrations) * }\end{array}$ & \\
\hline
\end{tabular}

* Biofilm studies on vegetables with conventional methods was incubated for $24 \mathrm{hr}$. While, the testing time of the spectroscopic method depends on biofilm concentration. The high concentrations of biofilm on vegetables can be detected at that time. The low concentration of biofilm must be incubated for enhanced the signal. 


\subsection{E. coli biofilm formation by scanning electron microscope}

The morphology and structure of biofilm formation of E. coli was analysed by SEM (Figure $5)$. The result showed that biofilm density has been increased with the times. First state, the single cell of bacteria used flagellum for cohesion the vegetable surface (Figure 5B). Second stage, figure 5C showed the cells of bacteria divided and the E. coli cells created the EPS structure for the coherent of cells-surface and cell-cells. Final state, the bacteria cells in biofilm structure would be grown and maturated (Figure 5D and 5E) [12].
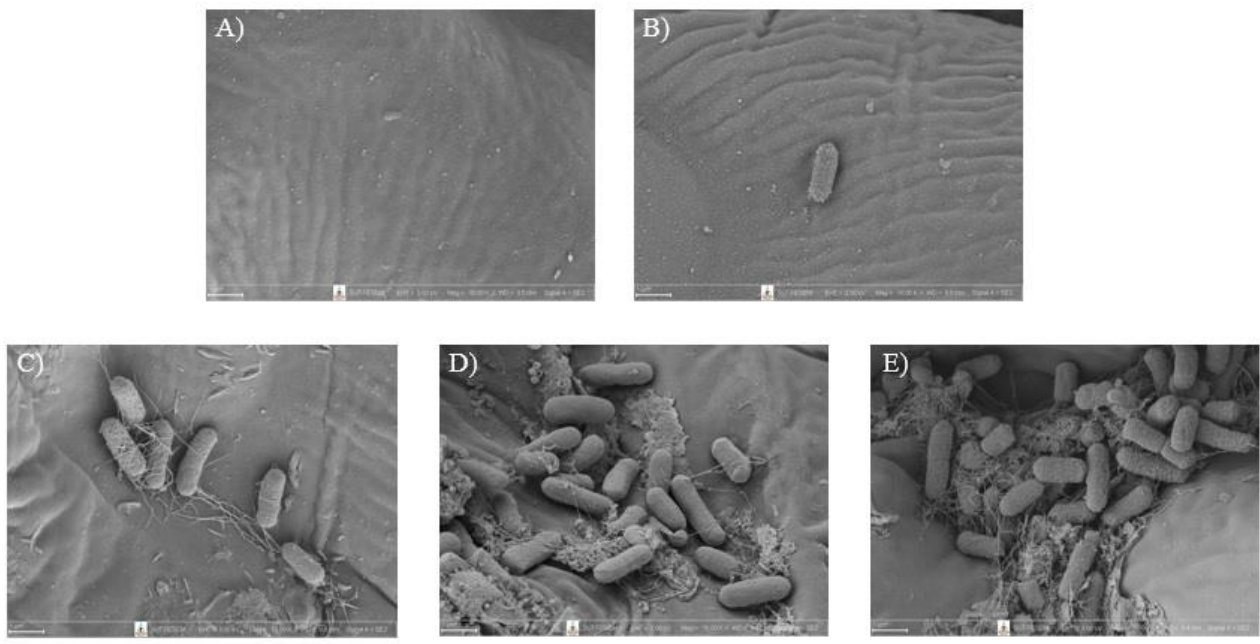

Fig. 5. E. coli biofilm formation on green oak salad by SEM. 10K Magnification A) control, B) After Incubated 30 minutes, C) Biofilm formation after incubated $8^{\circ} \mathrm{C} 24 \mathrm{hrs}$, D) $72 \mathrm{hrs}$, and E) $120 \mathrm{hrs}$.

The comparison results of SEM and CLSM of biofilm formation found that the autofluorescence of the green oak leaves was confirmed in 120x of CLSM and 1000 of SEM at day 5 . The most of cultures were attached to the leaves rather than stomata. Therefore, the response of red autofluorescence at $680 \mathrm{~nm}$ was the stomata area and the response of red autofluorescence at $620 \mathrm{~nm}$ was leaves. The result showed the concentration of autofluorescence at stomata area was relatively higher than at leaves.

\section{Conclusions}

The purpose of research has been applied spectral signal for detecting E. coli biofilm area on green oak salad. Green oak has a mainly responsive signal at 620 and $680 \mathrm{~nm}$. The contamination area was classified with a staining reagent emission spectrum. For this case, it is propidium iodide at $620 \mathrm{~nm}$ emission and the response of autofluorescence response in plant was reported to $680 \mathrm{~nm}$. The significance of signal intensity was not less than $90 \%$ for sample classification with and without $E$. coli biofilm. The results found that culture growing rate at storage condition $8^{\circ} \mathrm{C}$ has been decreasing when time has been increasing, but biofilm intensity directs variation with times.

This work was supported by Food Packaging and Innovation Research. The fund of National Research Council of Thailand and Equipment Analysis from Department of equipment, Suranaree University of Technology. 


\section{References}

1. M.R. Donlan, Emerging Inflections Diseases 8, 881-890 (2002).

2. O'Toole, A George, Journal of Visualized Experiments, 47(2011).

3. A.Bridier, F.D. Brissonnet, R. Briandet, Journal of microbiological methods volume 82, 64-70 (2010)

4. W.J. Kim, M.S. Kim, B.K Cho, Journal of Food Engineering 99, 314-322 (2010)

5. Chow goon Ng., Mun Fai Loke, Food Microbiology 62, $68-76$ (2017)

6. G.H. Krause, E. Weis, Annu.Rev. Plant physiol. Plant Mol Biol 42, 313-349(1991)

7. K. Kodama, Plos one. 2016

8. C. D. Everard, M.S. Kim, H.Y Lee. Journal of Food Engineering 143, 139 - 145 (2014)

9. N.C. de Melo, L.A Beijo, Amazonnian Journal of Agricultural and Environment Sciences 56, 80-88 (2013)

10. Sami Ullah Qadir, Vaseem Raja, Weqar A. Siddiqui. Ecotoxicology and Environmental Safety 129, 320-328 (2016)

11. S.P Pragyan, U. Chaudhary, S.K. Dube, Indian Journal of pathogen\&microbiology 59(2), 177-179 (2016).

12. S. Liu, C. Gunawan, N. Barraud, Environmental Science \& Technology 50, 8954-8975 (2016.) 